\title{
Interconverting Models of Gray Matrix and Geographic Coordinates Based on Space Analytic Geometry
}

\author{
Ni Zhan", Liang Lin, Ling Lou \\ College of Science, Guilin University of Technology, Guilin, China \\ Email: "zhanni105@163.com
}

Received November 5, 2012; revised December 5, 2012; accepted December 13, 2012

\begin{abstract}
With the progress and development of science, the synchronous satellite as the high-tech product is taken something more and more seriously by all countries. Converting gray value matrix into geographic coordinates becomes more and more important. This paper establishes the models for interconverting row and column of gray value matrix which detected by the infrared detectors on synchronous satellites and geographic coordinates based on the related theorems and properties of space analytic geometry. We draw satellite cloud image with the data of gray matrix in MATLAB, convert coastline's latitude and longitude coordinates into row and column coordinates of a gray matrix according via these models when the sub-satellite point's row and column coordinates and geographic coordinates are given, then add coast-lines to the original satellite cloud image with the transformation data. We find that coast-lines completely match with the bump characteristics of satellite cloud image, and that verify the correctness of the models.
\end{abstract}

Keywords: Space Analytic Geometry; Satellite Cloud Image; Geographic Coordinates; Gray Value Matrix; Coastline

\section{Introduction}

Satellite cloud image plays an important role in the scientific research of mastering atmospheric circulation, medium-and long-term weather forecast and disastrous weather and so on. It is drawn with grayscale data which converted from the temperature data over the Earth detected by infrared detector on geosynchronous satellite. It captures the distribution of clouds in the atmosphere, to find the weather system and verify the correctness of surface weather maps that have been drawn. Sub-satellite point represented by geographic latitude and longitude is the point at which a line between the satellite and the centre of the Earth intersects the Earth's surface. Infrared detector scans sampling in accordance with stepping angle (north-south direction) and row scanning angle. The satellite probe data is a gray value matrix, each element of the matrix corresponds to a sampling point of the Earth or extraterrestrial. Established the models that interconvert non-negative elements of grayscale matrix and geographic coordinates, taking up north down south left west right east as the rule, is not only a way to estimate the distance between each probe point, but also a method to the add coast-lines to a satellite cloud image accurately. In meteorological research and service, it is often posi-

\footnotetext{
*This research was supported by the Guangxi Natural Science Foundation of China under the Grant No. 2011GXNSFA018149.

\#Corresponding author.
}

tioning and reading the value of geographic coordinates in satellite cloud image, the low resolution and small image make positioning accuracy in a low level. Therefore it produces positioning error, which has an influence on forecast accuracy and research accuracy. Calculating the conversion formulas have attracted much attention, in particular among people interested in the remote sensing technology and meteorology.

Zhang Qingshan [1] uses linear regression to estimate satellite spin axis vector in the nephogram imaging process, calculating the exact position and attitude of the satellite on the basis of geographic coordinates, transforming the nephogram coordinates to geographic coordinates. Wang Guanghui \& Chen Biao [2] use the method of rotating spherical coordinates system to calculate longitude and latitude of the 2048th sampling point in scanline scanning by High Resolution Infrared Radiation Sounder. Zhang Jinjin \& Liang Hongyou [3] design algorithms whose objective is to converse plane coordinates to latitude and longitude coordinates use of IDL. Dong Minglun \& Zhou Jiong [4] educe the relationship between Cartesian conversions and Geographic conversions according to visual imaging principle of synchronous meteorological satellite. George P. Gerdan \& Rodney E. Deakin [5] review and analyze six published techniques of Cartesian-to-geographic conversion, which are regarded as representative of the numerous published methods, found that Lin \& Wang's method is appreciably 
faster than the other five methods.

In this paper, we do some research in conversion methods which could transform the row and column coordinates corresponding to gray value matrix into latitude and longitude coordinates, apply the geometry knowledge and ellipsoidal coordinates to establish conversion models, finally verify the models with the measured data consists of gray value matrix and latitude and longitude coordinates of coastline. This method which may be of use to practitioners is simple and feasible. The innovation of this paper is setting parameters for the sub-satellite point's latitude and longitude coordinates to establish models by using related theorems of space analytic geometry, makes the models more general, suitable for wherever the sub- satellite point located. Since the models have analytical solution, it improves the accuracy and is a relatively simple method.

The paper is organized as follows. Section 2 is the main part of this paper, converting coordinates models are investigated. Section 3 presents a numerical example to show the applications of the proposed methods. Section 4 concludes this paper with a brief summary.

\section{Converting Coordinates Models}

In order to converse row and column coordinates $(i, j)$ from geographic coordinates $(\lambda, \varphi)$, one important thing is to ensure the corresponding latitude and longitude of each element in gray value matrix. We consider the Earth as an ideal ellipsoid, which obtain through an ellipse rotates around the line connecting the north and the south poles. The semi-major of the ellipsoid is equatorial radius, semi-minor axes is polar radius. We take eighth of ellipsoid to create a three-dimensional coordinate system for research.

$X, Y, Z$ 3-D coordinates have an origin at the centre of the ellipsoid as shown in Figure 1. The $X-Y$ plane is the equatorial plane of the ellipsoid (the origin of latitudes), the $Z$-axis is in the direction of the rotational axis of the ellipsoid of revolution, the $Y$-axis is in the direction of the line connecting the infrared detector on geosynchronous satellite and the centre of the ellipsoid, which intersects with Earth's surface at sub-satellite point $(Q)$, and the $X$-axis is advanced $90^{\circ}$ west along the equator. The distance between the infrared detector and the centre of the ellipsoid is $O S$ line remarked as $c . P$ is an arbitrary sampling point on the Earth. The point $E$ is referred to as the projection of $P$ in the $X$-Y plane.Likewise, $L$ is the projection of $P$ in the $Y$ - $Z$ plane and $M$ is the projection of $P$ in the $X-Z$ plane. Drop a perpen- dicular EF from E to $X$ axis, and $E H$ is the perpendicular to $Y$ axis through the point $E, P M, P L$ are the perpen- diculars to $X$ axis and $Y$ axis through the point $P$, respec- tively.

The sampling point's row and column coordinates in the gray matrix can be expressed as $P(i, j), 3-\mathrm{D}$ coordinates expressed as $P(x, y, z)$, latitude and longitude coordinates expressed as $P(\lambda, \varphi), \lambda$ and $\varphi$ represent longitude and latitude of sampling point respectively. Thus sub-satellite point's row and column coordinates can be expressed as $Q(I, J)$, latitude and longitude coordinates expressed as $Q\left(\lambda_{0}, 0\right)$. Referring to Figure $\mathbf{1}$, the following relationships will be of use in the derivations that follow.

$$
\begin{gathered}
x=O F=E H, \quad y=O H=E F, \quad z=O G=P E, \\
\angle P O E=\varphi, \angle H O E=\lambda_{0}-\lambda, \quad \boldsymbol{O P}=r
\end{gathered}
$$

The stepping angle of infrared detector is $\alpha . O Q=a$ and $O N=b$ are the semi-major and semi-minor axes of the ellipsoid. Let $k$ and $l$ represent the total number of row scanning and column scanning, respectively, which count from $Q$ to $P$. Correspondingly, the detector turns respectively $k \alpha$ and $l \alpha$ radians in the direction of horizontal and vertical.

An ellipsoid is a closed quadric surface that is a three dimensional analogue of an ellipse. The standard equation of an ellipsoid centered at the origin of a Cartesian coordinate system is

$$
\frac{x^{2}}{a^{2}}+\frac{y^{2}}{a^{2}}+\frac{z^{2}}{b^{2}}=1
$$

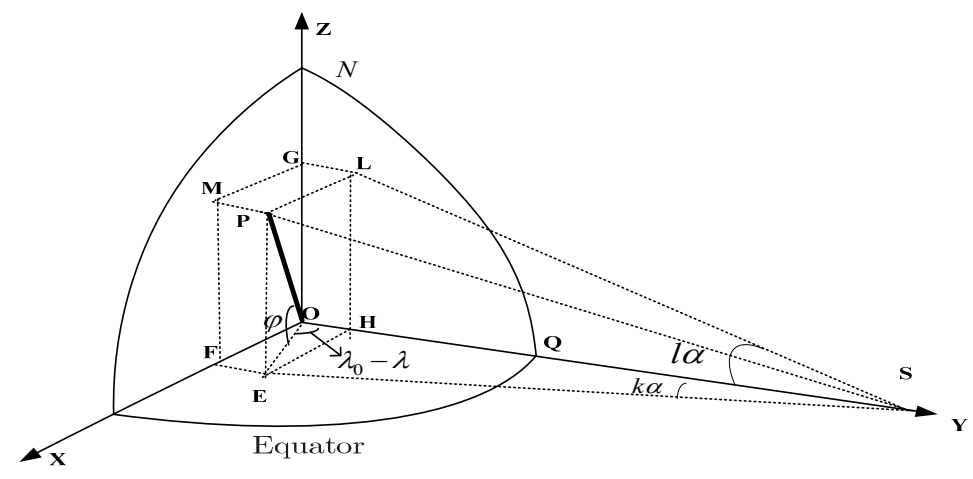

Figure 1. Section of ellipsoid. 
The surface of the ellipsoid may be parameterized in several ways. One choice which singles out the " $z$ "-axis is:

$$
\left\{\begin{array}{l}
x=r \cos \varphi \sin \left(\lambda_{0}-\lambda\right) \\
y=r \cos \varphi \cos \left(\lambda_{0}-\lambda\right) \\
z=r \sin \varphi
\end{array}\right.
$$

From right-angled triangle EHS we get:

$$
\tan (k \alpha)=\frac{x}{c-y}
$$

From right-angled triangle LHS we get:

$$
\tan (k \alpha)=\frac{x}{c-y}
$$

Build equations:

$$
\left\{\begin{array}{l}
x=(c-y) \tan (k \alpha) \\
z=(c-y) \tan (l \alpha) \\
y=a \sqrt{1-\left(\frac{x^{2}}{a^{2}}+\frac{z^{2}}{b^{2}}\right)}
\end{array}\right.
$$

From Equations (2) and (3) we work out:

$$
\left\{\begin{array}{l}
k=\frac{1}{\alpha} \arctan \left(\frac{r \cos \varphi \sin \left(\lambda_{0}-\lambda\right)}{c-r \cos \varphi \cos \left(\lambda_{0}-\lambda\right)}\right) \\
l=\frac{1}{\alpha} \arctan \left(\frac{r \sin \varphi}{c-r \cos \varphi \cos \left(\lambda_{0}-\lambda\right)}\right)
\end{array}\right.
$$

where $r=\frac{1}{\left(\frac{\cos \varphi}{a}\right)^{2}+\left(\frac{\sin \varphi}{b}\right)^{2}}$.

In this paper, we take sub-satellite point as the initial point to compute the row and column coordinates of the four cardinal directions: north, east, south and west. Therefore, we divide the ellipse into four quadrants taking sub-satellite point as origin, and discuss in four cases to converse $(k, l)$ to $(i, j)$. In the first quadrant, the formula can be:

$$
\left\{\begin{array}{l}
i=I+k \\
j=J-l
\end{array}\right.
$$

when $i>I, j<J$.

Put (4) into (5), that is:

$$
\left\{\begin{array}{l}
i=I+\frac{1}{\alpha} \arctan \left(\frac{a \cos \varphi \sin \left(\lambda_{0}-\lambda\right)}{c-a \cos \varphi \cos \left(\lambda_{0}-\lambda\right)}\right) \\
j=J-\frac{1}{\alpha} \arctan \left(\frac{a \sin \varphi}{c-a \cos \varphi \cos \left(\lambda_{0}-\lambda\right)}\right)
\end{array}\right.
$$

when $i>I, j<J$.

Similarly, we can get the other three quadrants' formulas:

The second quadrant:

$$
\left\{\begin{array}{l}
i=I-\frac{1}{\alpha} \arctan \left(\frac{a \cos \varphi \sin \left(\lambda_{0}-\lambda\right)}{c-a \cos \varphi \cos \left(\lambda_{0}-\lambda\right)}\right) \\
j=J-\frac{1}{\alpha} \arctan \left(\frac{a \sin \varphi}{c-a \cos \varphi \cos \left(\lambda_{0}-\lambda\right)}\right)
\end{array}\right.
$$

when $i<I, j<J$.

The third quadrant:

$$
\left\{\begin{array}{l}
i=I-\frac{1}{\alpha} \arctan \left(\frac{a \cos \varphi \sin \left(\lambda_{0}-\lambda\right)}{c-a \cos \varphi \cos \left(\lambda_{0}-\lambda\right)}\right) \\
j=J+\frac{1}{\alpha} \arctan \left(\frac{a \sin \varphi}{c-a \cos \varphi \cos \left(\lambda_{0}-\lambda\right)}\right)
\end{array}\right.
$$

when $i<I, j>J$.

The fourth quadrant:

$$
\left\{\begin{array}{l}
i=I+\frac{1}{\alpha} \arctan \left(\frac{a \cos \varphi \sin \left(\lambda_{0}-\lambda\right)}{c-a \cos \varphi \cos \left(\lambda_{0}-\lambda\right)}\right) \\
j=J+\frac{1}{\alpha} \arctan \left(\frac{a \sin \varphi}{c-a \cos \varphi \cos \left(\lambda_{0}-\lambda\right)}\right)
\end{array}\right.
$$

when $i>I, j>J$.

The four Formulae (6)-(9) above are the models that converse longitude and latitude coordinates into row and column coordinates.

Conversion from geographic coordinates to row and column coordinates is a simple operation. Inverse computations, on the other hand, are a little complex, now we want to use $(i, j)$ to deduct $(\varphi, \lambda)$. Consider the case of the second quadrant for example: put the first two equations of (3) into the third equation of (3), we have:

$$
(c-y)^{2}\left[\frac{\tan ^{2}(k \alpha)}{a^{2}}+\frac{\tan ^{2}(l \alpha)}{b^{2}}\right]+\frac{y^{2}}{a^{2}}=1
$$

Let $\frac{\tan ^{2}(k \alpha)}{a^{2}}+\frac{\tan ^{2}(l \alpha)}{b^{2}}=d$, we get $(c-y)^{2} d+\frac{y^{2}}{a^{2}}=1$, the solutions to this quadratic equation can be found directly from the quadratic formula. Solve for $y: y=\frac{a^{2} c d \pm a \sqrt{a^{2} d-c^{2} d+1}}{a^{2} d+1}$. As $y>0$, so we take $y=\frac{a^{2} c d+a \sqrt{a^{2} d-c^{2} d+1}}{a^{2} d+1}$.

Build equations: 


$$
\left\{\begin{array}{l}
x=(c-y) \tan (k \alpha) \\
z=(c-y) \tan (l \alpha) \\
\varphi=\arctan \frac{z}{\sqrt{x^{2}+y^{2}}} \\
\lambda=\lambda_{0}+\arctan \frac{x}{y}
\end{array}\right.
$$

Let $\tan (k \alpha)=m, \tan (l \alpha)=n$, from (10) we obtain $y=\frac{a^{2} c d+a \sqrt{a^{2} d-c^{2} d+1}}{a^{2} d+1}$, put it into (11), we get

where

$$
m=\tan ((I-i) \alpha), \quad n=\tan ((J-j) \alpha) \quad d=\frac{m^{2}}{a^{2}}+\frac{n^{2}}{b^{2}} .
$$

The other three equations can be obtained by the same method. We do not list all the cases in this paper.

\section{A Numerical Example}

In this section, we present our experiment results. It may be helpful to illustrate these models in a constructed example simple enough that an analytical approach is possible. The results reported here are based on $2288 \times 2288$ gray value matrix detected by a satellite detection which is located at $0^{\circ} \mathrm{N}$ latitude and $86.5^{\circ} \mathrm{E}$ longitude, that is $Q\left(\lambda_{0}, 0\right)=Q(86.5,0)$. Given the distance between satellite and the center of Earth is $42,164,000 \mathrm{~m}$, that is $c=$ $42164000 \mathrm{~m}$. The detection scans by the stepping angle of 140 micro-arcs, that is $\alpha=0.00014^{\circ}$. Given $a=$ $6378136.5 \mathrm{~m}, b=6356751.8 \mathrm{~m}$.

We select arbitrarily 40 sampling points on the column 1145 to test the models. Use (7) to convert the sampling points' row and column coordinates into longitude and latitude coordinates, then we want to use the results to calculate the row and column coordinates via (6)-(9), results as follow:

From Table 1 it can be seen that after converting the row and column coordinates into geographical coordinates, then converting back, there exists error of 1 - 3 pixels between the row coordinates before and after conversion in nine of the sampling points. The rest coordinates are wholly consistent. The error of $1-3$ pixels is rounding error which falls within the acceptable range. And results proved that the models are reasonable.

In reference [4], the calculated results exists 0.3 pixel error between the rows and 4.6 pixel between the columns, the models of this paper based on previous studies have higher accuracy.

Draw satellite cloud image with the data of gray value matrix in MATLAB [6], as shown in Figure 2(a), add coast-lines into satellite cloud image, shown in Figure 2(b).

Convert the data of coastline's longitude and latitude

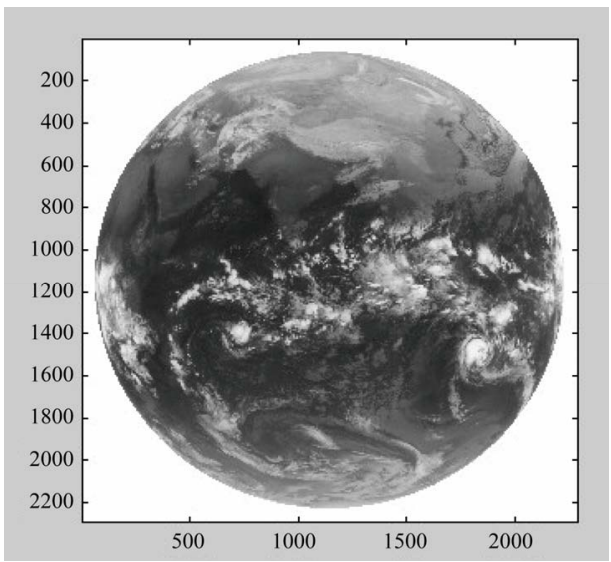

(a)

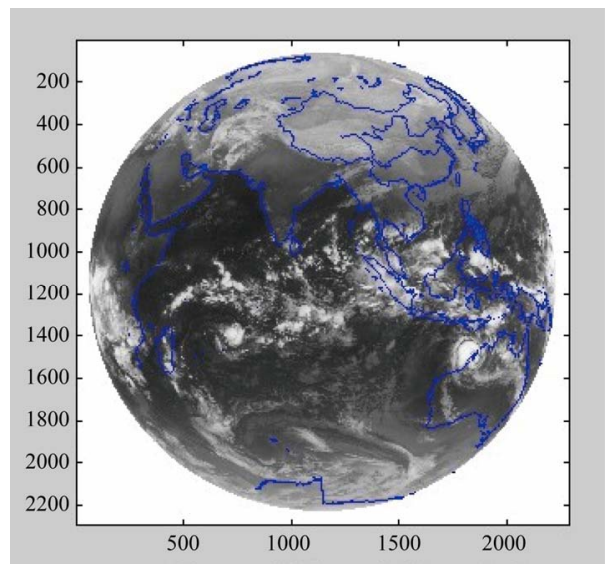

(b)

Figure 2. (a) Satellite cloud image; (b) Satellite cloud image with coast-lines.

$$
\left\{\begin{array}{l}
\varphi=\arctan \frac{n\left[c-\frac{a^{2} c d+a \sqrt{a^{2} d-d c^{2}+1}}{a^{2} d+1}\right]}{\sqrt{m^{2}\left(\frac{c-a \sqrt{a^{2} d-d c^{2}+1}}{a^{2} d+1}\right)^{2}-\left(\frac{a^{2} c d+a \sqrt{a^{2} d-d c^{2}+1}}{a^{2} d+1}\right)^{2}}} \\
\lambda=\lambda_{0}+\arctan \frac{m\left(c-a \sqrt{a^{2} d-d c^{2}+1}\right)}{a^{2} c d+\sqrt{a^{2} d-d c^{2}+1}}
\end{array}\right.
$$


Table 1. Results of converting coordinates.

\begin{tabular}{|c|c|c|c|c|c|}
\hline Row & Column & Longitude & Longitude & Row after converting & Column after converting \\
\hline 100 & 1145 & 86.5 & 65.82926 & 101 & 1145 \\
\hline 230 & 1145 & 86.5 & 49.9728 & 233 & 1145 \\
\hline 342 & 1145 & 86.5 & 41.1886 & 345 & 1145 \\
\hline 455 & 1145 & 86.5 & 33.91336 & 457 & 1145 \\
\hline 566 & 1145 & 86.5 & 27.61518 & 567 & 1145 \\
\hline 599 & 1145 & 86.5 & 25.85278 & 600 & 1145 \\
\hline 650 & 1145 & 86.5 & 23.2053 & 651 & 1145 \\
\hline 675 & 1145 & 86.5 & 21.93694 & 676 & 1145 \\
\hline 697 & 1145 & 86.5 & 20.83494 & 697 & 1145 \\
\hline 700 & 1145 & 86.5 & 20.68563 & 700 & 1145 \\
\hline 713 & 1145 & 86.5 & 20.04117 & 713 & 1145 \\
\hline 742 & 1145 & 86.5 & 18.61763 & 742 & 1145 \\
\hline 750 & 1145 & 86.5 & 18.22815 & 750 & 1145 \\
\hline 761 & 1145 & 86.5 & 17.69476 & 761 & 1145 \\
\hline 790 & 1145 & 86.5 & 16.29969 & 790 & 1145 \\
\hline 813 & 1145 & 86.5 & 15.20387 & 813 & 1145 \\
\hline 829 & 1145 & 86.5 & 14.44661 & 829 & 1145 \\
\hline 844 & 1145 & 86.5 & 13.74017 & 844 & 1145 \\
\hline 856 & 1145 & 86.5 & 13.17732 & 856 & 1145 \\
\hline 895 & 1145 & 86.5 & 11.36085 & 895 & 1145 \\
\hline 903 & 1145 & 86.5 & 10.99046 & 903 & 1145 \\
\hline 922 & 1145 & 86.5 & 10.11349 & 922 & 1145 \\
\hline 930 & 1145 & 86.5 & 9.74532 & 930 & 1145 \\
\hline 950 & 1145 & 86.5 & 8.827475 & 950 & 1145 \\
\hline 976 & 1145 & 86.5 & 7.639261 & 976 & 1145 \\
\hline 986 & 1145 & 86.5 & 7.183597 & 986 & 1145 \\
\hline 998 & 1145 & 86.5 & 6.637687 & 998 & 1145 \\
\hline 1000 & 1145 & 86.5 & 6.54679 & 1000 & 1145 \\
\hline 1002 & 1145 & 86.5 & 6.455919 & 1002 & 1145 \\
\hline 1011 & 1145 & 86.5 & 6.047289 & 1011 & 1145 \\
\hline 1050 & 1145 & 86.5 & 4.281411 & 1050 & 1145 \\
\hline 1111 & 1145 & 86.5 & 1.530465 & 1111 & 1145 \\
\hline 1120 & 1145 & 86.5 & 1.125251 & 1120 & 1145 \\
\hline 1124 & 1145 & 86.5 & 0.945185 & 1124 & 1145 \\
\hline 1130 & 1145 & 86.5 & 0.67511 & 1130 & 1145 \\
\hline 1135 & 1145 & 86.5 & 0.450065 & 1135 & 1145 \\
\hline 1140 & 1145 & 86.5 & 0.22503 & 1140 & 1145 \\
\hline 1145 & 1145 & 86.5 & 0 & 1145 & 1145 \\
\hline 1150 & 1145 & 86.5 & -0.22503 & 1149 & 1145 \\
\hline 1151 & 1145 & 86.5 & -0.40506 & 1152 & 1145 \\
\hline
\end{tabular}


into row and column coordinates, then add coast-lines into satellite cloud image. We can discover that coastlines exactly match with the bump characteristics of satellite cloud image. It illustrates the models are reasonable.

\section{Conclusions}

This paper has presented the methods with analytical solution of interconverting row and column of gray value matrix and geographical coordinates based on the related theorems and properties of space analytic geometry, finally gives acceptable results of a numerical example to verify the correctness of the models.

This work can also be easily applied in satellite image registration and so on. Our future work involves using these models in cloud motion models developed at satellite cloud image and analyzing its feasibility.

\section{REFERENCES}

[1] Q. S. Zhang, "Method of Static Meteorological Tatellite
Cloud Image Landmark Navigation," China Academic Journal Electronic Publishing House, Beijing, 1984, pp. 14-20.

[2] G. H. Wang, B. Chen and W. P. He, "Research of Longitude and Latitude of AVHRR 2048 Based on Polar Coordinate Transformation," Journal of Qingdao University, Vol. 16, No. 3, 2003, pp. 45-48.

[3] J. J. Zhang, H. Y. Liang, X. F. Chen, X. F. Gu, T. Yu and H. B. Ge, "Transformation from Image Panel Coordinate to Latitude and Longitude Coordinate," Geospatial Information, Vol. 8, No. 1, 2010, pp. 63-66.

[4] M. L. Dong and Q. Zhou, "Transforming Relationship between Cartesian Coordinates and Geographical Coordinates and Error Analysis," Marine Forecasts, Vol. 28, No. 2, 2011, pp. 48-54.

[5] G. P. Gerdan and R. E. Deakin, "Transforming Cartesian Coordinates $X, Y, Z$ to Geographical Coordinates $\varphi, \lambda, h$," The Australian Surveyor, Vol. 44, No. 1, 1999, pp. 55-63.

[6] D. Teng, P. Wang, Y.-G. Che and D. Wang, "Realization Method of Electronic Chart Based on Coaseline with MATLAB7.0," Laser Journal, Vol. 31, No. 5. 2010, pp. 10-11. 